\title{
RAS Division Model Evaluation Database Tools
}

\author{
Kevin T. Foster \\ Regional Atmospheric Sciences Division \\ Lawrence Livermore National Laboratory \\ Livermore, California 94551 USA
}

September 1994

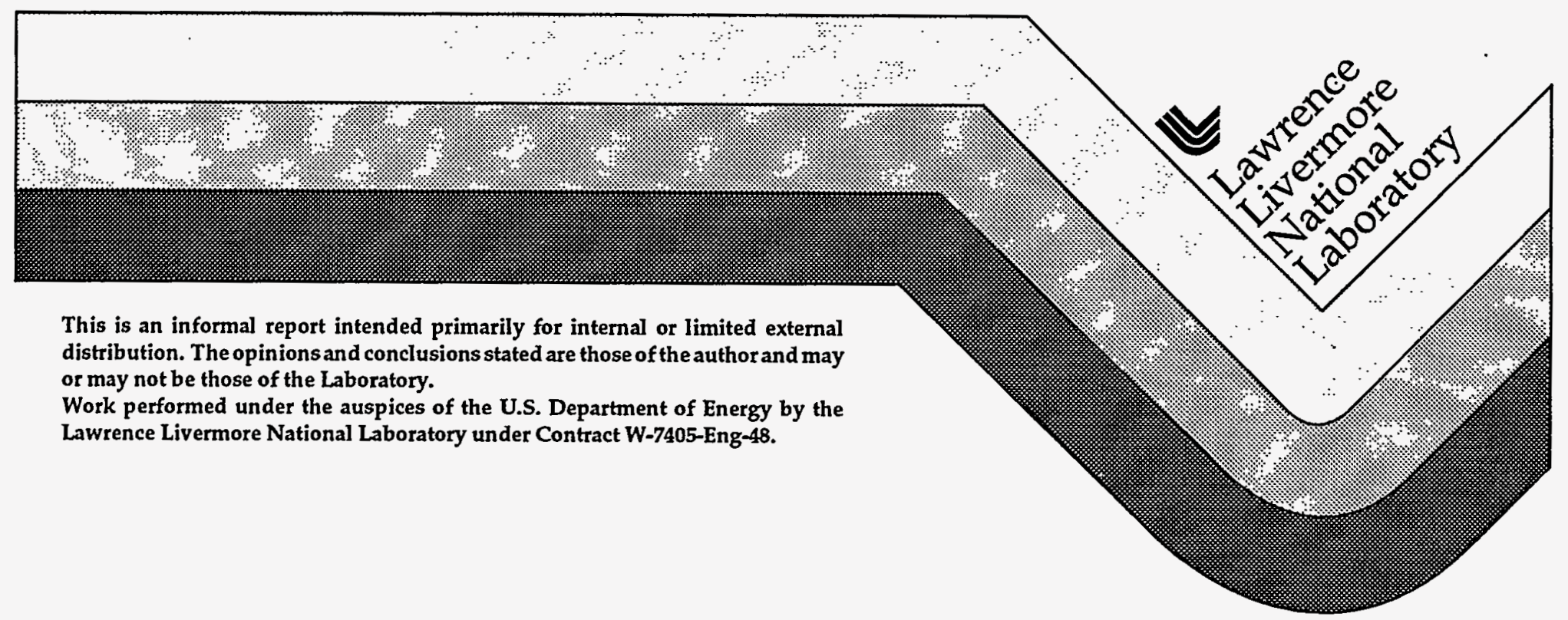




\section{DISCLAIMER}

This document was prepared as an acccount of work sponsored by an agency of the United States Government. Neither the United States Government nor the University of California nor any of their employees, makes any warranty, express or implied, or assumes any legal liability or responsibility for the accuracy, completeness, or usefulness of any information, apparatus, product, or process disclosed, or represents that its use would not infringe privately own rights. Reference herein to any specifie commercial products, process, or service by trade name, trademark, manufacturer, or otherwise, does not necessarily constitute or imply its endorsement, recommendation, or favoring by the United States Government or the University of California. The views and opinions of authors expressed herein do not necessarily state or reflect those of the United States Government or the University of California, and shall not be used for advertising or product endorsement purposes.

This report has been reproduced directly from the best available copy.

Available to DOE and DOE contractors from the Office of Scientific and Technical Information

P.O. Box 62, Oak Ridge, TN 37831

Prices available from (615) 576-8401, FTS 626-8401

Available to the public from the

National Technical Information Service

U.S. Department of Commerce

5285 Port Royal Rd.

Springfield, VA 22161 


\section{DISCLAIMER}

Portions of this document may be illegible in electronic image products. Images are produced from the best available original document. 


\section{RAS Division Model Evaluation Database Tools}

Kevin Foster

September 1, 1994

\section{Introduction}

Recently the RAS Division Model Evaluation Database (MEDb) was implemented. This database is primarily designed to hold atmospheric and tracer concentration data collected as part of various atmospheric field experiments. To allow users of this data to interface with the MEDb a series of computer tools have been developed. This document contains a discussion of these tools as they currently exist. The user is cautioned that these tools will likely evolve and expand as development resources become available and as the needs and requirements of the RAS Division staff are further defined. Before reading the remainder of this document it is recommended that the reader review the accompanying RAS Division Model Evaluation Database Description document for a detailed description of the MEDb, and for definition of some of the terms used in this document.

To date, MEDb tool development has focused on allowing the user to view, modify, add to, delete from, and generate hard copy of selected portions of the MEDb. To accomplish this, the SAPIENS Vision product has been used to create a Motif user interface to the VAX/RMS files which form the MEDb. This implementation is now available to users on the Division's ALPHA VAX systems. A corresponding implementation on the Division's VAX systems was completed earlier using SAPIENS' SmartStar product. Although the user may access this earlier SmartStar implementation by following the instructions in this document while logged into a VAX node, it is highly recommended that users initiate the tools on an ALPHA system and use the more complete Vision implementation. This document contains a detailed description of this Motif version only.

This document first presents a discussion on initiating the MEDb tools, followed by a description of common user interaction for all MEDb Motif forms. The remaining sections contain a detailed description of the MEDb editing interface and generation of MEDb hard copy. User's wishing to reformat MEDb wind velocity data for input into the MEDIC model should use these hard copy generation tools to create the needed input to the Format_MEDIC_Input program which is discussed in the accompanying Format MEDIC Input document. A similar discussion is presented for the Format_TIMEHIS_Input program (which reformats MEDb concentration data into a "Sampler.Dat" file) in the accompanying Format TIMEHIS Input document. Appendix A contains figures depicting the Motif windows used to interface with the MEDb. 


\section{Initiating MEDb Tools}

The MEDb tools are initiated through the use of six or nine character mnemonics. (These mnemonics were named following the rules governing the naming of the ARAC production account mnemonics.) To define these mnemonics the user must execute the DCL command file "dsk\$modev_root: [tools.commands]setup_modev.com", where "dsk\$modev_root" is a system logical. Once this is done the following mnemonics (symbols) are defined:

$\begin{array}{ll}\text { EdiDat } & =\text { Edit Dataset } \\ \text { EdiMeL } & =\text { Edit Metdata Libraries } \\ \text { EdiScD } & =\text { Edit Scalar Data } \\ \text { EdiSou } & =\text { Edit Sources } \\ \text { EdiTrL } & =\text { Edit Tracer Libraries } \\ \text { EdiWid } & =\text { Edit Wind Data } \\ \text { RepMeLSen } & =\text { Report Metdata Library - Sensors } \\ \text { RepMeLSta } & =\text { Report Metdata Library - Stations } \\ \text { RepTrLSen } & =\text { Report Tracer Library - Sensors } \\ \text { RepTrLSta } & =\text { Report Tracer Library - Stations } \\ \text { RepScD } & =\text { Report Scalar Data } \\ \text { RepWiD } & =\text { Report Wind Data } \\ \text { ShoDaD } & =\text { Show Dataset Directories } \\ \text { StaFMI } & =\text { Start Format MEDIC Input } \\ \text { StaFTI } & =\text { Start Format TIMEHIS Input } \\ \text { StaPST } & =\text { Start Plot Stats TIMEHS } \\ \text { StaPIS } & =\text { Start Plot Stats }\end{array}$

(Use of the last two mnemonics are discussed in the Plot Stats User's Guide.) Note that users should not execute the setup_modev command file from an ARAC Production or BETA account because of potential conflicts with Production/BETA mnemonics.

\section{Show Dataset Directories (ShoDaD):}

Many of the mnemonics listed above will initiate a form providing the user with an interface to one or more MEDb files. Depending upon the type of file(s) being accessed by a given form, the initiating mnemonic may require either zero, one, or two accompanying arguments which will indicate the directory and filename(s) of the target file(s). These arguments and the files accessed by each of the mnemonics are discussed in the following sections.

The mnemonic ShoDaD will provide the user with directory listings of the appropriate master MEDb directories. Entering "ShoDaD" displays a directory listing of the processed MEDb dataset directories where the names of the directories are (dataset ID).DIR. Entering "ShoDaD (dataset ID)" displays a directory listing of all Atmospheric and Tracer Measurement files contained in the dataset identified by the dataset ID. These Measurement files are named as (measurement type)_(modifier).DAT. (These terms are described in the accompanying RAS Division Model Evaluation Database Description document.) These dataset ID and measurement type_modifier strings will be needed as mnemonic arguments. 
The user may restrict the directory listing of Measurement files by entering any portion of the target measurement type or modifier string as a second argument to ShoDad. For example, entering "ShoDaD dopptex air" will generate a directory listing of the DOPPTEX AirTemp, AvgAirCcn_Freon, and AvgAirCon_SF6 Measurement files.

\section{Common MEDb Motif Interaction}

For the purposes of this document, the user is assumed to have a basic familiarity with the Motif Graphical User Interface. User's can assume standard Motif interaction if not otherwise specified. This section briefly reviews the common features of the MEDb Motif forms, and some of the user interaction conventions used. It is split into sub-sections which contain information on fields, scrolling areas, and buttons common to the forms discussed in this document. Users are referred to the SAPIENS' Vision documentation for a more detailed discussion of these features.

\section{Fields:}

Enterable text fields are distinguished as dark rectangular areas typically opposite or under the field's associated text label. There is no field value checking other than that done to verify a match to how the field was defined during form creation (e.g. numerals for date/time fields, limit on number of enterable characters for a given field, etc.). All non-numeric fields will typically allow any enterable character, regardless of whether the entered string is a valid MEDb entry (e.g. Use Flag values other than $Y$ or $N$ ). The user will notice that alphabetic characters entered for values of keyed components (Station ID and Dataset Name) in the MEDb RMS files will be shifted to upper case. This is to assure proper linkage among all appropriate MEDb records.

Field labels do not usually include formatting or units information for the associated field. This information may be displayed for a given field by pressing the Help key when the field has focus. The user is cautioned that while most formatting and units questions will be answered by this field help, not all fields currently have associated help.

Upon new.record creation, all empty (null) fields for the record will display default values. These values are blanks for non-numeric fields, -99999 for values representing integers, and -99999.0 for values representing floating point numbers. User's may modify these values at any time, as with any enterable field. Negative (Positive) values may be changed to positive (negative) by pressing the $+(-)$ key while in any portion of the numeric field.

\section{Scrolling Regions}

There are three types of scrolling regions used in the MEDb forms. The first region is an enterable scrolling field for Comments which are part of an $\mathrm{MEDb}$ record. This scrolling field accepts any characters and will "word-wrap" at the right field boundary: It may be scrolled only in the vertical direction. 
The second type is a non-enterable message scrolling field (later referred to as the "Message Field"). This is identified as a rectangular scrolling area near the bottom of the form of the same color as the form's background. Application messages and field help strings are displayed in this field. (User's should be aware that if multiple messages are generated as the result of a users interaction, each individual message overwrites the previously displayed message. This results in only the last generated message of the series displayed and accessible to the user.) The area may be scrolled both in the horizontal and vertical.

The third type is a scrolling region containing numerous fields which display the contents of "detail" records (e.g. the Station Sensor records are "detail" records in the Model Evaluation Station and Sensor Libraries form). These fields scroll as a group of fields on each line of the scrolling region. Each group (i.e. line) displays the contents of a single "detail" record. ("Detail" is a term used by the Vision product documentation to emphasize a record's logical many-to-one relationship with its "master" record. For example, a single Station Library record (the "master") is associated with several Sensor Library records (the "details") since a given station often has many sensors.) These regions may be identified as a large, lightly colored rectangular areas containing several columns and rows of enterable fields, and may be scrolled both horizontally and vertically. Note that a horizontal scroll will not scroll the associated column labels.

\section{Buttons:}

There may be up to eleven buttons on the MEDb forms. Three deal specifically with detail record scrolling regions and will be discussed first. These buttons will be in close proximity to the scrolling detail record region (where one exists) and are labeled, More Details, New Detail, and Delete Detail.

As the user retrieves master MEDb records, a maximum number (currently set to 30 ) of associated detail records are retrieved at any one time. If more detail records exist than have been retrieved and displayed in the detail scrolling area, the user is notified of this in the Message Field. The user may then repeatedly click the More Details button to retrieve an additional sets of records which are added to those already displayed in the scrolling area. When all detail records have been retrieved and displayed, the user is notified of this in the Message Field and the More Details button is desensitized (grayed out).

User's wishing to delete or add detail records from the MEDb will use the Delete Detail or Add Detail buttons, respectively. Clicking the Delete Detail button will eliminate the detail record which has focus from the scrolling area, and will "mark" the record for deletion. Clicking the New Detail button will insert a line of blank fields in the scrolling region immediately below the line having focus (or at the end of the scrolling list if no scrolling line has focus). Users may then enter appropriate values for the new record. Once added to the database, display of the new record(s) during future retrievals from the database will place the records at the end of the scrolling list. Note that the records will not actually be deleted or added to the associated database until the user presses the Save button. 
The Save button is one of the eight remaining buttons used on the MEDb forms which typically run the width of the form just above the Message Field. No database additions, deletions, or modifications are made until the user clicks the Save button. Clicking the Save button will process the changes to only the currently retrieved master record and its associated detail records. This means that users must click the Save button to process any changes to the currently displayed record(s) prior to retrieving another master record. Once another master record is retrieved all previous changes are lost if the Save button was not pressed.

The effects of clicking the Delete button are quite different than clicking the Delete Detail button. Clicking the Delete button will "mark for delete" ALL detail records associated with the currently displayed master record whether they have all been retrieved or not (if there is a detail scrolling region on the form), along with marking the displayed master record for deletion. A click of the Save button will complete the deletion process.

Users wanting to add a new master record (i.e. records which have no fields in a scrolling region such as a Measurement or Station Library record) should click the New button. This will clear all form fields. After the user enters the appropriate field values, clicking Save will create the new record in the database. Note that in the Model Evaluation Sources form clicking New will also add a new detail record in the detail scrolling region. In other forms containing detail scrolling regions the user must add the first detail record of a new master by separately clicking the New Detail button.

Users may undo additions, deletions, and modifications to the currently displayed record(s) since the last press of the Save button by clicking the Reset button. Reset will return the record to its original state at the time it was last saved.

The Qualify, Next, and Previous buttons allow the user to retrieve selected database records. Clicking Qualify clears all fields and displays a single blank detail record (for forms having a detail record scrolling area). Users may then enter values for any combination of master or detail fields that they wish to have govern future record retrievals. (Users will notice that the formatting characters ("/", "@", and ":") disappear from the date/time fields in qualify mode. In the current Vision implementation users must enter qualifying dates and times as a 12-character string with no formatting characters.) These values may be expressed as exact matches, partial matches, non-matches, or value ranges. Users should refer to the Vision documentation for a complete list of options. To retrieve records which have an exact match for a specific field value users should simply enter the desired value into the appropriate field. For convenience a few of the more often used qualifier symbols are listed here for some of the other ways to qualify a field:

* used as a wild card for character strings ("air*" retrieves any records with the field value beginning with "air"),

!= or ! used to negate an expression ("!=air*" retrieves any records in which the field does not begin with."air"), 
null value ("!= " retrieves records in which the field contains a nonnull value),

$>\quad$ greater than (">12.2" retrieves records in which the field value is greater than 12.2),

$<\quad$ less than,

$>=$ greater than or equal to,

$<=$ less than or equal to,

\& and ("> $12.2 \& !=20.0$ " retrieves any record in which the field value is greater than 12.2 but is not equal to 20.0 ),

, or (" $<=0.0,>360.0$ retrieves any record in which the field value is negative or is greater than 360 ),

... inclusive range ("10...20" retrieves any record in which the field value is greater than or equal to 10 and less than or equal to 20 ).

Once the user has entered the appropriate qualifier(s), if any are desired, he may use the Next and Previous buttons to retrieve records which match any currently defined qualifiers. Records will be retrieved in order of the value for the primary key (Dataset Name for the Model Evaluation Datasets form, Station ID for the Model Evaluation Station and Sensor Libraries form, and Measurement Ending Time for the Wind Velocity Data form and Scalar Data form). Users will be notified in the Message Field when there are no more records which match the defined qualifiers. If no qualifiers have been entered, records are retrieved from the beginning of the database (lowest primary key value). The current qualifier values are cleared by clicking the Qualify button.

The final button is the Close button. Clicking Close clears the form from the screen and returns the user to DCL prompts.

As the user executes various database functions using the buttons, he can verify his current functional "mode" by referring to the immediate right of the Message Field. A single word will appear to tell him if he is in "Update" mode (able to make modifications to the displayed record(s)), "Insert" mode (is currently entering field values for a new record), or "Qualify" mode (is currently entering qualifier field values). Some buttons which would initiate incorrect actions while in certain modes will be desensitized when appropriate.

\section{MEDb Editing Tools}

This section pertains to the MEDb tools which allow the user to view and make changes to the files which comprise the MEDb. These tools are initiated with the six character Edi-- mnemonics listed in the previous section. These mnemonics may be used on either the VAX or ALPHA VAX systems. As mentioned earlier however, users are strongly encouraged to run the MEDb editing tools from an ALPHA system which will provide a Motif user interface. This interface gives complete access to all MEDb variables, whereas the nonMotif VAX implementation provides limited variable access and data manipulation features. Only the Motif interface is described here. 
Users of these Editing Tools will not have access to the master MEDb files. Instead, upon entry of an Edi-mnemonic, a search of the user's current directory is done to find the needed file(s). If the files are not in the directory, a copy of the appropriate master MEDb file(s) is created in the user's current directory. A message is displayed to the user indicating whether the files were found in the current directory, or if a copy of the master files is being created. In this way a user may maintain a local modified version of the master MEDb files. (Note that users should not execute the editing tools while in the MEDb dataset : directories. File protection restrictions will prevent the tools from operating while in these directories.)

Any desired permanent changes to the master files should be forwarded to the MEDb Coordinator. To assist the user in deleting an unwanted copy of these files from his current directory, a prompt for deletion of any file copied during the execution of the mnemonic will 'appear after the user closes the associated Motif window. These prompts will appear only when a file has been copied into the current directory during the mnemonic execution, and not if the needed files already exist locally. Users may also delete the local files using the $\mathrm{DCL}$ delete command at any time without affecting the master MEDb files. It is the user's responsibility to properly manage local copies of the MEDb.

\section{Edit Dataset (EdiDat):}

Entering the mnemonic "EdiDat" provides access to the General Dataset and Dataset Measurement Type Information files. No arguments are required for this mnemonic. Figure A-1 illustrates the Motif user interface initiated by this mnemonic.

The upper section of the form, plus the Comments area near the bottom, allow users access to the each of the General Dataset records (one record at a time). The scrolling area in the middle of the form displays the Dataset Measurement Type Information records associated with each retrieved General Dataset record. Records are linked through the Dataset Name field which is a . component of each of the two types of records.

\section{Edit Sources (EdiSou):}

Entering the minemonic "EdiSou" provides access to the Dataset Source Term Information file. No arguments are required for this mnemonic. Figure A2 illustrates the Motif user interface initiated by this mnemonic.

The two top fields on the form show Dataset Source Term Information record component values common to all records displayed in the scrolling area below. Each click of the Next button actually retrieves and displays all source term records for a single experiment (shown in the Source Name field) of a dataset.

Because the relationship between the scrolled and non-scrolled data fields of this form is quite different from the field relationships in other forms with scrolling areas, users may note slightly different interaction and display functionality. Whereas other form scrolling areas contain data which are stored in files with different record structures from the data displayed in the non- 
scrolled fields, the data displayed in the non-scrolled fields of this form come from the same record as scrolled data. This distinction should not be important to the casual user, but may explain minor interaction aberrations.

\section{Edit Metdata Libraries (EdiMeL) and Edit Tracer Libraries (EdiTrL):}

Entering the mnemonics "EdiMel (dataset ID)" or "EdiTrL (dataset ID)" provides access to the meteorological or tracer Measurement Station and Sensor Information Library files, respectively, for the dataset identified by the dataset ID. The dataset ID is a required argument. Figure A-3 illustrates the Motif user interface initiated by this mnemonic.

The upper section of the form, plus the Comments area near the bottom, allow users access to the each of the dataset's Measurement Station Library records (one record at a time). The scrolling area in the middle of the form displays the Measurement Sensor Library records associated with each retrieved Station Library record. Records are linked through the Station ID field which is a component of each of the two types of records.

\section{Edit Scalar Data (EdiScD):}

Entering the mnemonic "EdiScD (dataset ID) (measurement type_modifier)" provides access to the Atmospheric or Tracer Measurement file indicated by the dataset ID and measurement type_modifier combination. (Do not include the ".DAT" extension as part of the second argument, and do not use this mnemonic for WndSpdDir files.) Both arguments are required. Figure A-4 illustrates the Motif user interface initiated by this mnemonic.

This form retrieves and displays a single Measurement file record at a time. Users must realize the Measurement Type and associated Units being accessed by this form to correctly interpret the Measurement field value.

\section{Edit Wind Data (EdiWiD):}

Entering the mnemonic "EdiWiD (dataset ID) (measurement type_modifier)" provides access to the Atmospheric Measurement wind velocity file (WndSpdDir) indicated by the dataset ID and measurement type_modifier combination. (Do not include the ".DAT" extension as part of the second argument, and do not use this mnemonic for any Measurement files other than WndSpdDir files.) Both arguments are required. Figure A-5 illustrates the Motif user interface initiated by this mnemonic.

This form retrieves and displays a single Measurement file record at a time. Currently, wind velocities are the only MEDb Measurement Type stored as a vector quantity. If additional vector Types are stored, this form may be generalized to handle any vector quantity (similar to the generalized scalar data form discussed above).

\section{MEDb Hard Copy Generation Tools}

Users may generate ASCII disk files in their current directory which may then be printed for hard copy of the MEDb data. This is done through the use of 
the Rep--(-) mnemonics. As with the Edi--- mnemonics, if the appropriate $\mathrm{MEDb}$ file(s) are resident in the user's current directory they are used as the source of the data to be printed. If however they are not in the current directory, the master MEDb files are directly accessed. No files are ever copied into the ${ }^{y}$ user's current directory during the use of Rep--(--) mnemonics. Since these mnemonics do not allow changes to the accessed data files there is no danger of accidental corruption of the master files. The user is notified whether the process is accessing a local current directory file, or is accessing the "global" master MEDb files.

Note that there are no public hard copy tools available to print contents of the "high-level" data stores (General Dataset Information, Dataset Measurement Type Information, and Dataset Source Term Information). Hard copies of the appropriate portions of each of these database files exist with each dataset's filed documentation. See the MEDb Coordinator for this information.

As of this writing, the Rep-(--) mnemonics (which use the SAPIENS SmartReport product) only work from a VAX node. It is expected however that SmartReport will soon be licensed on the ALPHA VAX nodes which will permit use of these mnemonics on the ALPHAs.

Report Metdata Library (RepMeL--) and Report Tracer Library (RepTrL--):

Hard copy files of the four libraries (Metdata Station, Metdata Sensor, Tracer Station, and Tracer Sensor) for a given dataset are generated with the mnemonics RepMeLSta, RepMeLSen, RepTrLSta, and RepTrLSen, respectively. Each of these mnemonics requires a dataset ID as an argument (e.g. "RepTrLSta mats" would generate a hard copy file for the MATS dataset tracer station library).

Upon entry of one of these mnemonics, the user is notified that the report is being created and displays the file specification of the ASCII file. These files are created in the user's current directory and will be named "met_stations.lis", "met_sensors.lis", "tracer_stations.lis", or "tracer_sensors.lis" depending upon the mnemonic in use. These files will contain the entire contents of the library file (except for the Comments field for the Station Library records), and may be inspected by a file editor or sent to any printer for hard copy.

\section{Report Scalar Data (RepScD) and Report Wind Data (RepWiD):}

Like the Rep-mnemonics, RepScD and RepWiD use the SmartReport product to generate properly formatted ASC!I files. However because users will typically wish to print only portions of an MEDb Measurements file, the procedure for generating wind or scalar measurement data hard copy is a bit more involved.

These two mnemonics require a dataset ID and a measurement type_modifier as arguments (e.g. "RepScD dopptex AvgAirCcn_SF6" will access DOPPTEX dataset average SF6 air concentration data). After entering the mnemonic and associated arguments, a SmartStar (non-GUI) form will appear. If users wish to generate a hard copy of the entire MEDb file, they should now press the <esc> 
key followed by the keyboard $8 \mathrm{key}$. This will generate the corresponding ASCII file in the current directory which will be named "(measurement type_modifier).lis".

This will however typically generate very large files since all measurement data in the accessed file will be formatted for output. To reduce this amount of data, users should first press the $\langle e s c\rangle$ key followed by the keyboard 7 key. This puts the user in a field qualify mode. The user can then move from field to field (using the arrows or Return key) and press the keypad period (.) to enter qualifying field values. All qualifier symbols listed earlier will work except for the logical "and" ("\&") and the logical "or" (","). Users may replace the comma for logical "or" with a backslash ("/"). After entering all desired qualifiers the user should then press the $\langle e s c>-8$ key sequence. Only data records meeting the qualifier value restrictions will be placed in the ASCII disk file. (Users may press an $\langle e s c>-0$ key sequence to exit the form without generating an ASCII disk file.)

As an example, if the user wishes to restrict the hard copy to data with ending measurement times between January 1,1994 at 12:00 to January 2, 1994 at 3:00 (UTC), inclusive, he should enter "94/01/01@12:00:00...94/01/02@03:00:00". (The "/", "@", and ":" formatting characters are automatically inserted.) After typing the qualifier value, press the Return key to complete the value's entry.

Users needing to generate the wind measurements data file for input to the Format_MEDIC_Input program should follow this procedure using the RepWiD mnemonic. Once this has been done, the user may enter the StaFMI (Start Format MEDIC Input) mnemonic to execute the Format_MEDIC_Input program. (Refer to the accompanying Format MEDIC Input document for additional details.)

Similarly, users needing to generate the sample concentrations data file for input to the Format_TIMEHIS_Input program should follow this procedure, but use the RepScD mnemonic. (Refer to the Format TIMEHIS Input document.) 


\section{APPENDIX A \\ Sample MEDb Interface Forms}




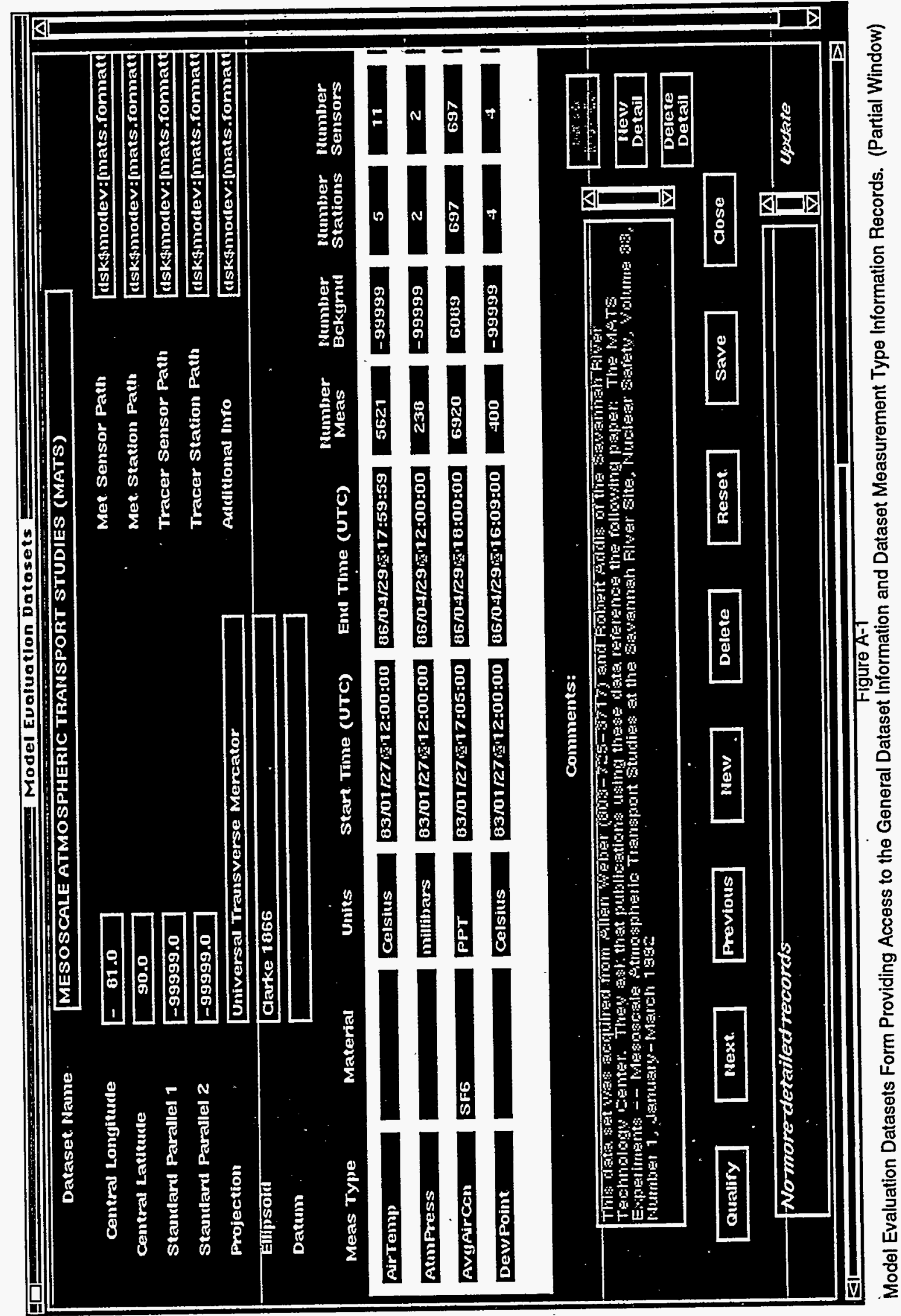




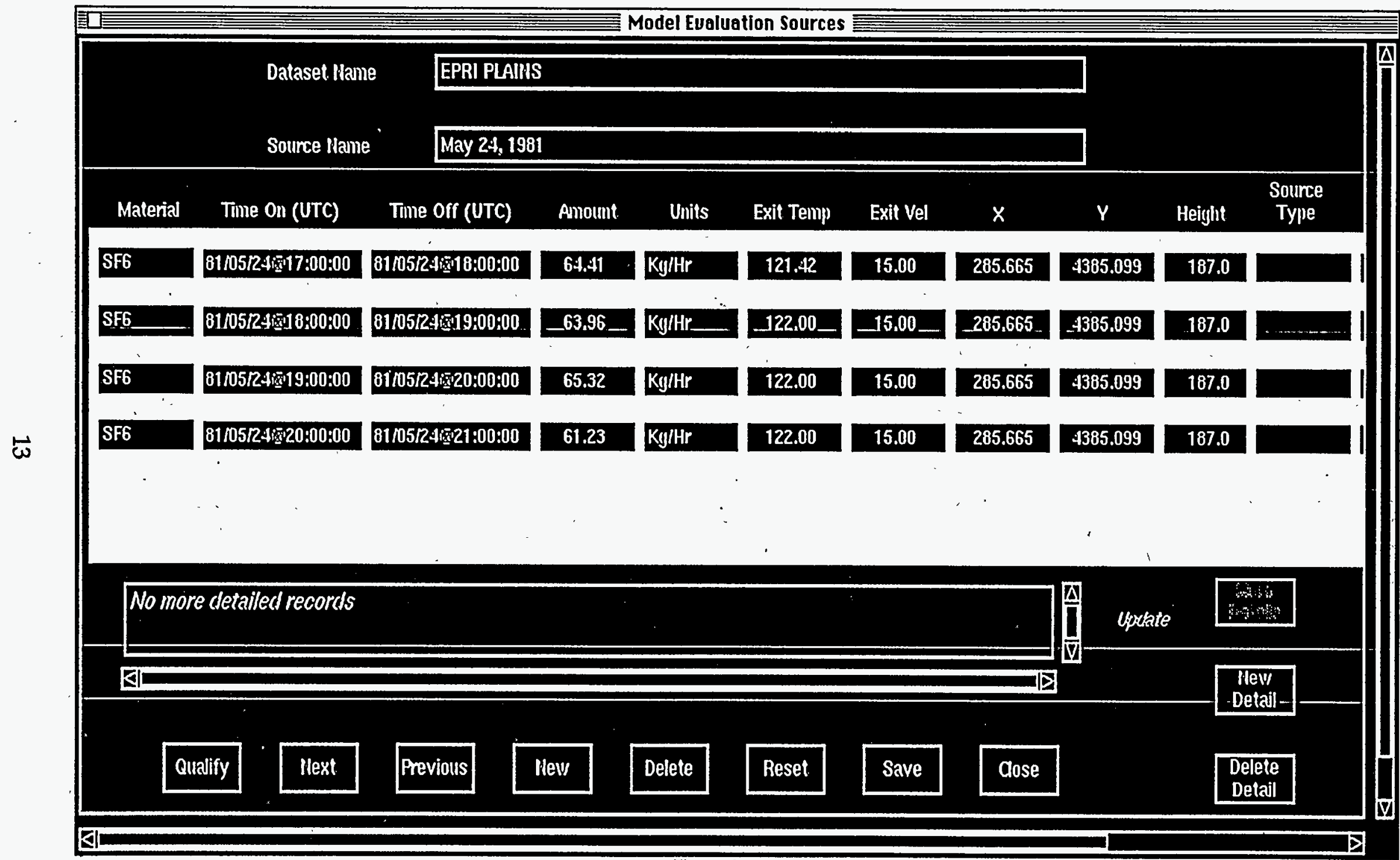

Figure A-2
Model Evaluation Sources Form Providing Access to the Dataset Source Term Infórmation Records. (Partial Window) 


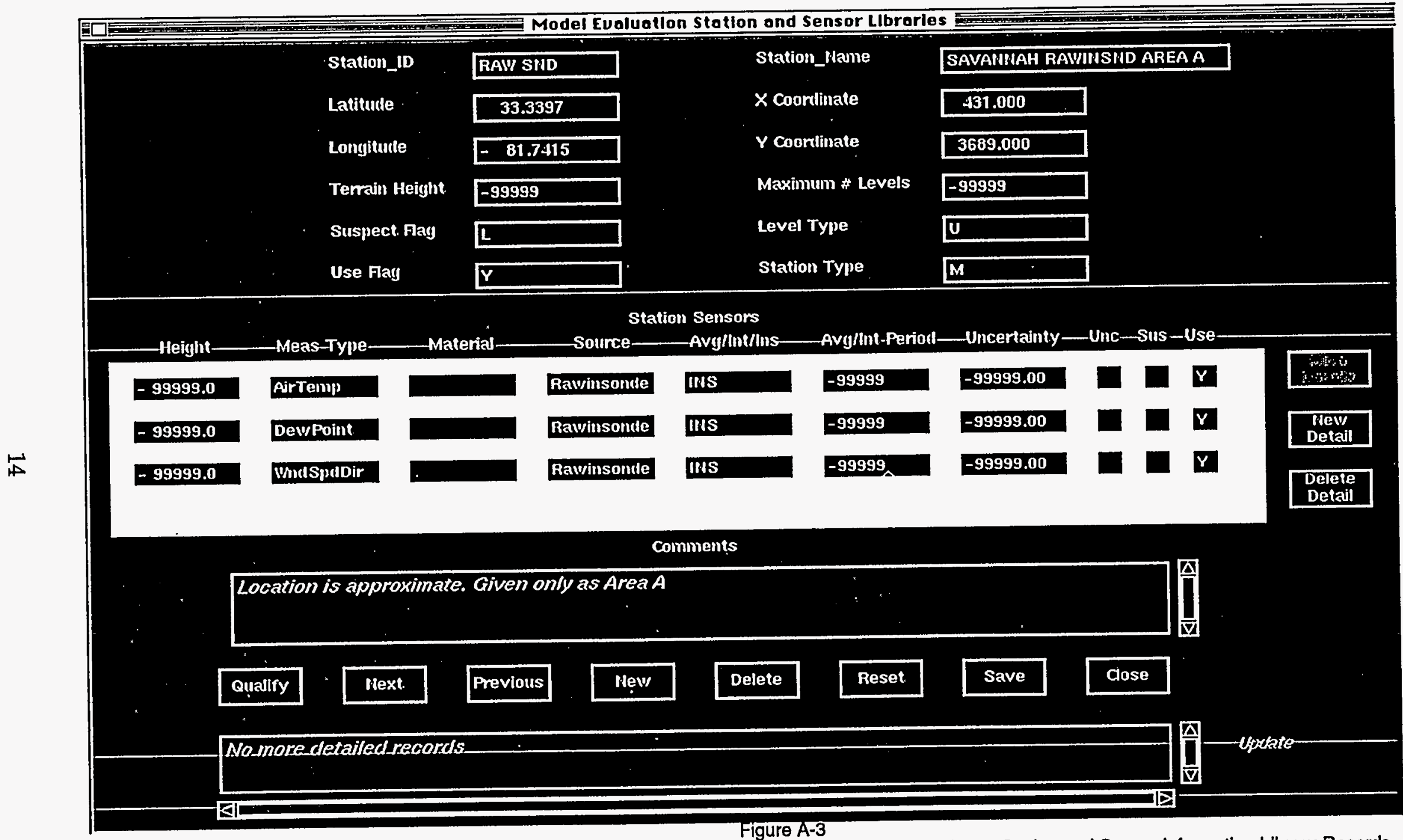

Model Evaluation Station and Sensor Libraries Form Providing Access to the Meteorological or Tracer Station and Sensor Information Llbrary Records. 


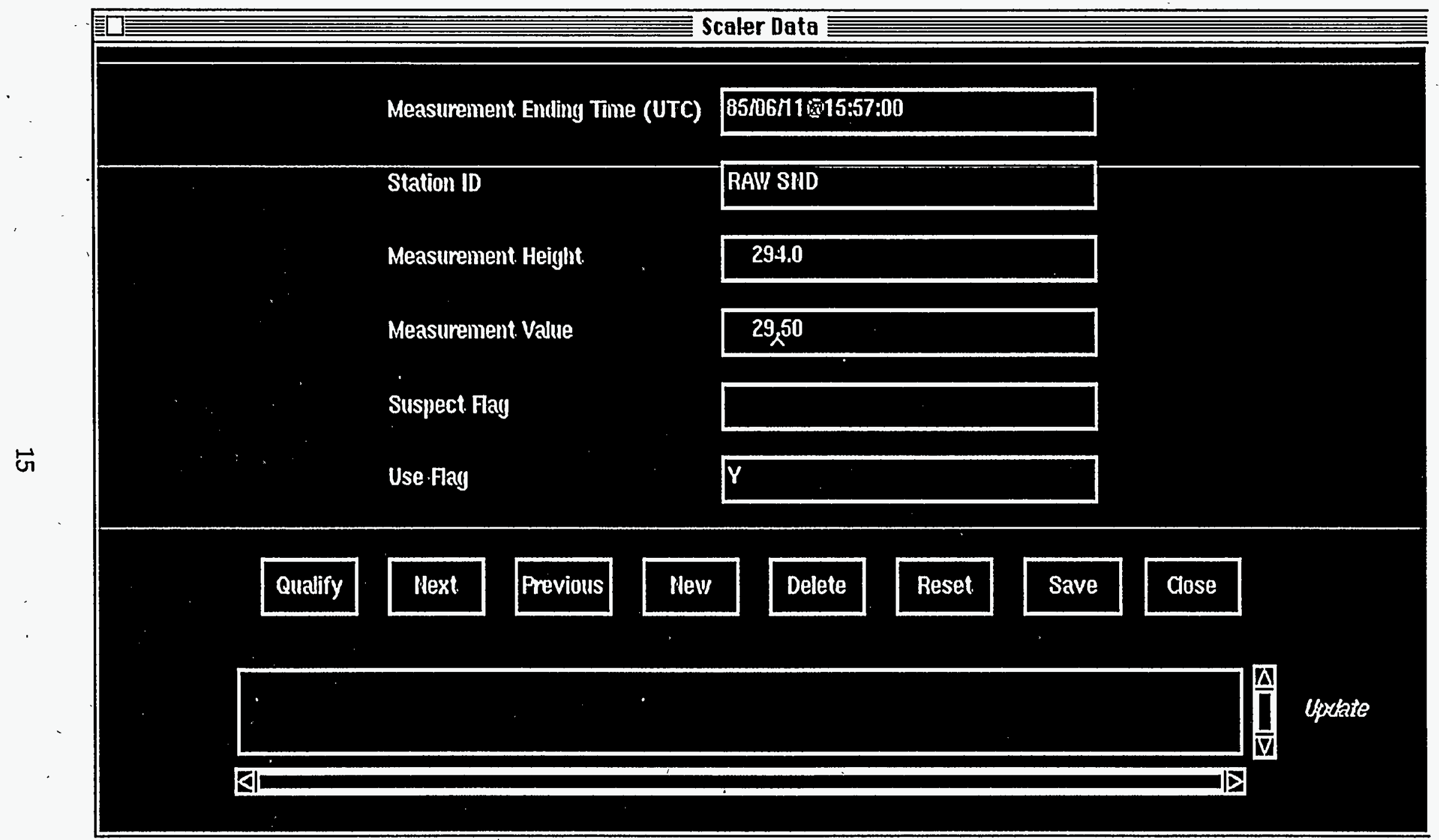

Figure A-4

Scaler Data Form Providing Access to the Tracer Measurements or Scaler Atmospheric Measurements Records. 


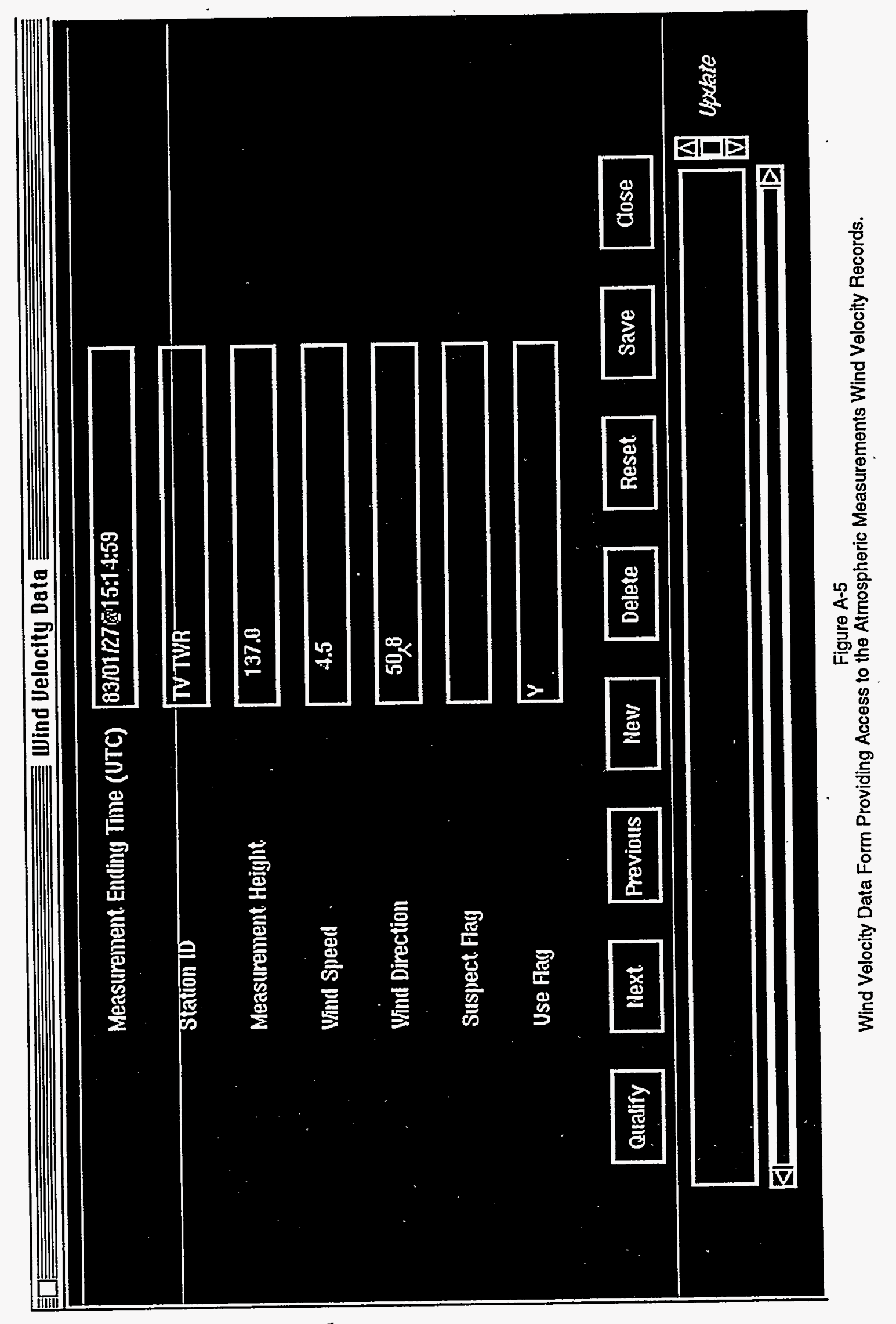

